\title{
Emerging Nanomaterials for Healthcare - An Interdisciplinary Conference at the University of Warwick
}

\author{
Mathew P. Robin *, Gemma-Louise Davies \\ Department of Chemistry, University of Warwick, Coventry, UK \\ *Correspondence: m.p.robin@warwick.ac.uk
}

\begin{abstract}
Emerging Nanomaterials for Healthcare", a one-day conference held at the University of Warwick on 28 November 2014, brought together over 80 academics, postgraduates and industrialists from 17 institutions and organisations from across the UK. The aim of the meeting was to provide an interdisciplinary forum to discuss research towards solving current problems in healthcare using "smart" treatments based on nanomaterials. In addition to stories of success, an emphasis was also placed on lessons learned, as well as visions for future directions in this rapidly expanding field. Seven speakers and over twenty poster presentations directed discussion throughout the day, while the meeting closed with an interactive panel discussion.
\end{abstract}

Keywords: Nanoparticles, nanomedicine, nanomaterials, healthcare, medicine

(C) Copyright: The Authors. This article is issued under the terms of the Creative Commons Attribution NonCommercial Share Alike License, which permits use and redistribution of the work provided that the original author and source are credited, the work is not used for commercial purposes and that any derivative works are made available under the same license terms.

\section{Emerging Nanomaterials for Healthcare - An Interdisciplinary Conference at the University of Warwick}

In a recent editorial for the journal Angewandte Chemie, Younan Xia asked the question 'Are we entering the Nano Era?' (Xia, 2014: 12268). Specifically, Xia was pondering whether we have reached the stage where materials fabricated with nanometre dimensions (between one and one hundred billionths of a metre) will begin to dominate technological advances in all areas of science. The importance of nanomaterials to the scientific community, and the impact of the nanotechnology that these materials enable, is a consequence of a unique set of features possessed by matter at the nanometre scale. As Stark et al. have recently noted: 'Valuable chemical industry products are a result of designing a set of properties within a material. Today, industrial development therefore considers nanoparticles as an extended 
toolbox containing traditionally difficult to realize properties' (Stark et al., 2015: 2). Despite the growing importance of nanotechnology to the areas of catalysis, pigments and composite materials, significantly less commercial success has so far been achieved for nanomaterials in healthcare. In his piece, Xia frames the driving forces for interest in nanotechnology applied to biomedical research in particular, saying 'cells are packed with complex, functional structures with at least one dimension on the nanoscale' (Xia, 2014: 12268) and therefore 'the power of nanomedicine lies in its ability to operate on the same molecular scale as the intimate biochemical functions involved in the growth, development, and aging of the human body' (Xia, 2014: 12269). However it is this power, derived from the complexity of the potential interactions between synthetic nanomaterials and intimate biological functions that has slowed the translation of nanomaterials to practical medical application. Not only do desirable modes of action need to be intimately understood, but the toxicity of nanomaterials must also be fully addressed.

The development of nanomaterials for healthcare is a particularly pressing question for the two of us (MPR and GLD), as we both hold Fellowships from the Institute of Advanced Study at the University of Warwick, focussed toward undertaking nanotechnology-based research in the Department of Chemistry. With this concept in mind, we aimed to organise a conference that would bring together researchers working to advance the use of artificial nanomaterials in healthcare applications giving rise to the event "Emerging Nanomaterials for Healthcare", which took place at the University of Warwick on 28 November 2014. One of the great challenges in this field is the number of traditional disciplines which the research typically spans; from the physical understanding of the properties of nanoparticles, to the chemistry of their fabrication, and the biology of their action as therapeutic species. For this reason, a multidisciplinary conference provided an ideal forum for the exchange of ideas and expertise, as well as an opportunity to develop the kind of collaborative research networks that are vital for success in this field. We chose to invite seven speakers whose expertise spanned a range of topics across synthetic chemistry, materials science and biology and whose research shares the common goal of understanding and developing tools to improve the diagnosis and treatment of disease for the betterment of human health.

Synthetic chemistry is often divided into two distinct disciplines; "organic chemistry" pertaining to man-made compounds and materials which primarily contain those elements found in natural organic matter (principally carbon, oxygen, hydrogen and nitrogen), and "inorganic chemistry" which investigates compounds based on metallic elements 
(for example iron, gold, cobalt and many more besides). Professor Nguyễn T.K. Thanh from University College London used her talk to highlight some of her research group's efforts to fabricate a wide variety of inorganic particles with sub-100 nm dimensions from metallic elements. Thanh demonstrated that her team have been able to achieve control over both the size and shape of particles (with spheres, cubes, rods, and even stars manufactured with incredible control over reproducibility) as well as variations in particle surface coatings and the layering of multiple metallic elements. Such metal nanoparticles have applications in medical diagnosis; as their magnetic character can allow them to function as contrast agents in magnetic resonance imaging (MRI), improving the detection efficiency and therefore the quality of the images produced. Thanh presented recent work where this tactic had been used to improve the study of neural stem cell engineering, with the ultimate target of achieving spinal cord reconstruction.

Dr Catherine Berry from the University of Glasgow works very closely with biological cell lines, with the aim of understanding how nanosized materials interact with human cells and tissues. She presented work which utilises magnetic metal nanoparticles for "magnetofection", whereby clinicians would be able to control the movement of nanoparticles through a patient's body towards the area of diseased tissue (such as a tumour) by using an external magnetic field. Research from the Berry group has focussed on coupling this concept with the use of cell penetrating peptides (naturally occurring signalling molecules that allow material to cross the cell membrane and enter cells) so that the metal nanoparticles could be directed exclusively to diseased cells and subsequently gain entry. Work in this field has the ultimate goal of eliminating the unpleasant side-effects of chemotherapy, which result from the non-specific action of cancer cell killing drugs thus resulting in the simultaneous death of cancerous and healthy cells during treatment. This strategy is a common theme in nanomedicine research, and indeed several other speakers presented a variety of approaches to achieve the goal of specificity in treatment.

In addition to using an external stimulus, the presence of certain biomolecules or the natural differences in the local environments between different areas of tissue and within cells can also be exploited as a trigger for directed drug delivery. In his talk, Dr Seb Spain from the University of Nottingham (now at the University of Sheffield) demonstrated how nanoparticles can be designed to respond to specific deoxyribonucleic acid (DNA) sequences. DNA is the building blocks of genes which contain the instructions for the development and function of living organisms, and DNA communicates these instructions by interacting with the biomolecules that make up the "machinery" of the 
cell. Crucial to Spain's research was the attachment of specific DNA sequences to polymers; synthetic organic materials that can be designed to mimic natural surfactants such as lipids (the main component of cell membranes) in their ability to assemble into agglomerates on the nanometre scale. The resulting hybrid DNA-polymer nanoparticles subsequently showed a response to complementary DNA, which could therefore allow them to use the over expression of certain DNA sequences (such as occurs in cancers) as a trigger for treatment specificity.

As well as being targeted as an indicator of disease, nucleic acids can also be utilised for therapy. The presentation from Dr David Fulton of Newcastle University highlighted his recent efforts to achieve the controlled delivery of nucleic acid therapeutic agents using polymer nanoparticles. The assembly of polymers into particles on the nanometre scale allows them to be loaded with a cargo, such as molecules of a drug compound, while forming a protective layer around this cargo to prevent unwanted clearance from the body by the immune system or the action of the kidneys. The motive for using this well-established technique in Fulton's research is the attempt to improve the circulation time within the blood (and therefore the potency) of cancer therapy which uses short interfering ribonucleic acids (siRNAs). These siRNAs serve to silence the action of a particular gene, for example those which are specific to the division and growth of cancer cells, therefore leading to selective death of only the diseased cancerous cells.

Also focussing on the use of polymer nanoparticles for drug delivery was Professor Marina Resmini from Queen Mary University of London.

Recent work from her group has concentrated on using nanoparticles for transdermal drug delivery, which are topical treatments that enter the body by crossing through the skin rather than by an oral or intravenous administration. This is typically a challenging mode of action for therapy, as the skin has evolved to provide a robust barrier against foreign bodies in order to protect internal tissue from toxic and pathogenic materials. The polymer nanoparticles developed by Resmini's team were labelled with a fluorescent dye, a material that absorbs light of higher energy (such as blue light) and emits light of a lower energy (such as green light). This fluorescent tag enabled the location of the nanoparticles to be tracked using confocal microscopy (which couples conventional light microscopy with fluorescence detection) following administration to the skin surface, allowing monitoring of the passage of the nanoparticles across the skin, and their ultimate distribution within the tissue below and hence a thorough understanding of their behaviour and potential as delivery agents. 
The challenge of advanced diagnosis was addressed by the final two speakers. Dr Matthew Gibson and Daniel Phillips from the University of Warwick both discussed how they have used hybrids of metal (gold) nanoparticles, surface coated with organic polymers to create sensors for diagnostic applications. In both cases the choice of gold nanoparticles was motivated by the fact that they can be manipulated to give an "easy to assess" visual readout. Due to an effect known as surface plasmon resonance, a solution of gold nanoparticles will change colour if the individual nanoparticles begin to agglomerate into clusters. Phillips exploited this feature to produce a colorimetric test for levels of ferrous iron ( $\mathrm{Fe}^{3+}$ ions), of interest due to the atypically high levels of $\mathrm{Fe}^{3+}$ linked to a number of diseases, such as Alzheimer's and Parkinson's. Ferrous iron sequestering units attached to a polymer immobilised onto the surface of gold nanoparticles led to particle agglomeration in the presence of high levels of $\mathrm{Fe}^{3+}$ ions, due to interparticle bridging. This resulted in the characteristic colour change from the gold nanoparticles, thus providing a simple readout for diagnosis. Gibson presented work that uses a similar approach for the detection of bacterial toxins. In this case, the gold nanoparticle's polymer shell can selectively bind to the toxin (a model for highly poisonous "ricin"), which again leads to particle agglomeration and the diagnostic colour change. As Gibson explained in the introduction to his talk, the use of gold nanoparticles in diagnostic tests is well established, with the Clearblue pregnancy test - arguably the words most ubiquitous diagnostic test - based on similar technology. In fact, as Xia points out "nano" is nothing new at all. Before it became a buzzword, people had already used nanomaterials for many decades, if not centuries' (Xia, 2014: 12268). Simple and versatile chemistry such as that based on gold nanoparticles will inevitably continue to find applications in emerging nanomaterials for healthcare.

The conference closed with our speakers participating in a lively panel discussion, fielding questions from the assembled delegates. Perhaps the most pressing issue raised was the importance of working in collaborative research groups. Despite the majority of the panel identifying as Chemists, there was a clear imperative to work in collaboration with research groups possessing expertise in Biology, Life Sciences, as well as clinicians and members of the pharmaceutical industry. In the words of one panel member, 'it is important to avoid inventing challenges' because they offer an opportunity to do interesting chemistry, only to find that a problem never existed in the first place. Instead, research should be directed by dialogue between the various disciplines to identify a clear need in the first instance. Another point on which the panel were agreed was the importance of testing materials in biologically relevant conditions early in their development. For example, 
a nanoparticle formulation intended as an intravenous treatment which is insoluble in blood has no future, and it is essential to discover this sooner rather than later. The panel discussion finished with the speakers suggesting advances and directions for research that they hope to see tackled in the near future. Answers included the desire for a standardised test for the efficacy of drug release from nanoparticle delivery agents to enable comparison of data obtained by researchers working on similar problems across the globe, and a desire for a more complete understanding of the whole body immune system response to nanoparticles, which must surely be required for nanomedicine to become a clinical and commercial success.

The conference "Emerging Nanomaterials for Healthcare" gave a great demonstration of the exciting possibilities for synthetic nanomaterials to be applied to solve problems in healthcare. Talks from our invited speakers, poster presentations from over twenty of the delegates, and the panel discussion illustrated the strength and depth of current nanomedicine research in the UK. We hope to see the field continue to expand in the coming years, with increasing numbers of nanomaterial therapies and diagnostics appearing in the clinic. As Xia concludes, 'only when this relatively new and still seemingly bizarre realm of nano is able to make a positive and long-lasting impact on every aspect of our society, can we finally declare the arrival of the nano era' (Xia, 2014: 12271).

We would like to extend our thanks to the conference sponsors:

Institute of Advanced Study, University of Warwick; Materials Global Research Priority, University of Warwick; Materials Chemistry Division, Royal Society of Chemistry; Nanoscale and Journal of Materials Chemistry $B$, Royal Society of Chemistry; the Polymer Club, University of Warwick; ATG Scientific.

More details and photographs from the "Emerging Nanomaterials for Healthcare" 2014 Meeting can be found at http://warwick.ac.uk/enh2014.

\section{References}

Xia, Y. (2014), 'Are We Entering the Nano Era?', Angew. Chemie Int. Ed., 53 (46), 12268-71

Stark, W. J.; Stoessel, P. R.; Wohlleben, W.; Hafner, A. (2015), 'Industrial applications of nanoparticles', Chem. Soc. Rev., DOI: 10.1039/c4cs00362d 


\section{To cite this article:}

Robin, M. P \& Davies, G. (2015). Emerging Nanomaterials for Healthcare An Interdisciplinary Conference at the University of Warwick. Exchanges: The Warwick Research Journal, 2(2), 321-327. Retrieved from: http://exchanges.warwick.ac.uk/index.php/exchanges/article/view/59 